\title{
Entero-encephalopathy due to FBXL4-related mtDNA depletion syndrome
}

\author{
Josef Finsterer ${ }^{\odot}$ \\ Klinik Landstrasse, Messerli Institute, Vienna, Austria.
}

With interest we read the article by Köse et al. ${ }^{1}$ about an 11-months-old Turkish male with entero-ecephalopathy due to the compound heterozygous variants c.772G $>\mathrm{T}$ (p.Gly258) and c.1061G >C (p.Trp354Ser) in FBXL4. The patient manifested clinically with dysmorphism, developmental delay, axial hypotonia, cerebral atrophy, intractable myoclonic epilpesy, optic atrophy, dysphagia, gastro-intestinal dysmotility, intestinal bleeding, and severe lactic acidosis. We have the following comments and concerns.

It is well-known that FBXL4 variants may cause secondary mtDNA depletion. ${ }^{2}$ Thus, we should know the mtDNA copy number in different tissues to assess if the phenotype correlated with tissue variations of the mtDNA copy number.

FBXL4 variants cause multiple respiratory chain complex dysfunction. ${ }^{3}$ Thus, we should know if biochemical investigations were carried out and which of the respiratory chain complexes showed reduced activity.

FBXL4 variants have been reported to manifest with recurrent infections. ${ }^{3}$ Since the patient had recurrent pneumonia we should know if immunological parameters (leukocyte counts, interleukines) were within normal limits or abnormal. Involvement of the immune-system in mitochondrial disorders (MIDs) has been previously reported. ${ }^{4}$ In this respect we should know if gastro-intestinal bleeding was due to

$\triangle$ Josef Finsterer fifigs1@yahoo.de

Received 17th August 2020, accepted 6th September 2021. infectious gastro-enteritis and if the patient experienced other recurrent infectious diseases.

The index patient had inherited one of the variants each from the mother and father respectively, and the brother had inherited only the variant from the mother. We should know if any of these mutation carriers was prospectively investigated and if any of them manifested clinically or subclinically with typical MID features.

Epilepsy was classified as intractable but only phenobarbital (PB), levetirazetam (LEV), vigabatrin (VGB), and topiramate (TPM) were tried. Mitochondrial myoclonic epilepsy may favourably respond to benzodiazepines. ${ }^{5}$ We should know if benzodiazepines were ever tried and why epilepsy was initially treated with a combination of three anti-seizure drugs (ASDs).

From some of the ASDs it is well-known that they are potentially mitochondrion-toxic and may exhibit severe side effects. Particularly, PB may exert adverse effect on respiratory chain functions. It is also conceivable that gastrointestinal compromise was increased due to the application of VGB as VGB can be associated with severe gastro-intestinal side effects.

Since FBXl4 variants may manifest with strokelike episodes (SLEs) ${ }^{6}$ of which epilepsy is a dominant feature, we should know the results of multimodal MRI after onset of epilepsy. In particular diffusion weighted imaging, apparent diffusion coefficient, perfusion weighted imaging, and oxygen-extraction MRI should be shown, to assess if epilepsy was associated with a stroke-like lesion. 
The authors mention that abdominal sonography was normal but show an abdominal X-ray with massive intestinal distension. The authors should explain this discrepancy and if it was attributable to different time points at which the investigations were carried out.

According to figure 1, facial dysmorphism also included downslanting palpebrae, hypertelorism, and broad nose root.

In summary, this interesting case report has a number of shortcomings and reveals some discrepancies which should be solved. We should know the degree of mtDNA depletion, the amount of respiratory chain complex dysfunction, if the cellular or humoral immune system was affected, and if the heterozygote mutation carriers manifested clinically or not.

Key words: mitochondrial, mtDNA, depletion, multisystem, epilepsy.

\section{REFERENCES}

1. Köse E, Köse M, Edizer S, et al. Different clinical presentation in a patient with two novel pathogenic variants of the FBXL4 gene. Turk J Pediatr 2020; 62: 652-656.

2. Wang S, Lin L, Wang Y, et al. Novel homozygous mutation in the FBXL4 gene is associated with mitochondria DNA depletion syndrome-13. J Neurol Sci 2020; 416: 116948

3. El-Hattab AW, Dai H, Almannai M, et al. Molecular and clinical spectra of FBXL4 deficiency. Hum Mutat 2017; 38: 1649-1659.

4. Finsterer J, Zarrouk-Mahjoub S. Affection of immune cells by a C10orf2 mutation manifesting as mitochondrial myopathy and transient sensory transverse syndrome. Acta Neurol Belg 2017; 117: 969-970.

5. Finsterer J, Scorza FA, Fiorini AC, Scorza CA. Mitochondrial myoclonic epilepsy requires specific treatment. Seizure 2020; 78: 168-169.

6. Ebrahimi-Fakhari D, Seitz A, Kölker S, Hoffmann GF Recurrent stroke-like episodes in FBXL4-associated early-onset mitochondrial encephalomyopathy. Pediatr Neurol 2015; 53: 549-550.

\title{
Reply \\ Different clinical presentation in a patient with two novel pathogenic variants of the FBXL4 gene
}

\author{
Engin Köse ${ }^{\odot}$ \\ Division of Pediatric Metabolism and Nutrition, Department of Pediatrics, Ankara University Faculty of Medicine, Ankara, Turkey. \\ enginkose85@hotmail.com
}

\section{Dear Editor,}

We read with interest the "letter to editor" entitled "Entero-encephalopathy due to FBXL4related mtDNA depletion syndrome" which evaluated the article written by Köse et al. ${ }^{1}$

The author asked about the biochemical investigations for the respiratory chain complexes activity. Although we agree with the reviewer that this should have been evaluated I am afraid we were unable to assess the respiratory chain complexes activity.
The author mentioned that FBXL4 variants have been reported to manifest with recurrent infections due to involvement of the immunesystem in mitochondrial disorder and gastrointestinal bleeding may be due to gastrointestinal infection. ${ }^{2}$ However, the stool findings of patient (stool culture, microscopic assessment) were unremarkable. Furthermore, laboratory parameters (white blood cell account, C-reactive protein) was not compatible with infection. With these findings, in this patient gastrointestinal infection was ruled out. 
Another issue brought up in this letter queried if any of the mutation carriers were prospectively investigated and if any of them manifested clinically or subclinically with typical mitochondrial disorders features. All parents and sibling were evaluated for the findings of mtDNA depletion findings. However, no clinical findings were revealed.

The author stated that Mitochondrial myoclonic epilepsy may favorably respond to benzodiazepines. ${ }^{3}$ We initiated benzodiazepine (midazolam) infusion for refractor epilepsy. However, a poor response was seen. Secondly, other antiepileptic drugs were initiated one by one. A ketogenic diet was tried for intractable epilepsy but it failed. At last, epilepsy was controlled with 4 antiepileptic drugs. Gastrointestinal symptoms and findings did not persist in the clinical follow-up except for intestinal dysmotility. In other words, no side effects of vigabatrin treatment were seen.

The results of the multimodal MRI after the onset of epilepsy was questioned. For the index patient, diffusion MRI was performed at 2 and 7 month-old-age. In both MRIs, no findings of stroke-like lesions were detected. We agree that this information should have initially been added and thank the author for this question.

We mentioned that abdominal sonography was normal but showed an abdominal X-ray with massive intestinal distension in the report. Abdominal sonography was performed to assess dysmorphological findings, the presence of intra-abdominal bleeding and to evaluate for acute abdomen. We detected a massive intestinal distension with X-ray (at the same time with abdomen sonography) and physical examination and in clinical follow-up intestinal dysmotility and distension persisted. However, gastrointestinal bleeding discontinued.

We agree with the author concerning the additional facial dysmorphism findings such as down slanting palpebrae, hypertelorism, and broad nose root.

We thank the author for their interest in our work and hope that we were able to sufficiently address all the questions.

Key words: mitochondrial, mtDNA, depletion, multisystem, epilepsy, FBLX4.

\section{REFERENCES}

1. Köse E, Köse M, Edizer S, et al. Different clinical presentation in a patient with two novel pathogenic variants of the FBXL4 gene. Turk J Pediatr 2020; 62: 652-656.

2. El-Hattab AW, Dai H, Almannai M, et al. Molecular and clinical spectra of FBXL4 deficiency. Hum Mutat 2017; 38: 1649-1659.

3. Finsterer J, Scorza FA, Fiorini AC, Scorza CA. Mitochondrial myoclonic epilepsy requires specific treatment. Seizure 2020; 78: 168-169. 